\title{
Comparación de valores de temperatura obtenidos con termómetro ótico digital y con termómetros axilar y rectal de mercurio en menores de 5 años
}

\author{
PETER MCCOLL C. ${ }^{1}$, KAREN COHEN SCH. ${ }^{2}$, FRANCISCA SOTO-AGUILAR B. ${ }^{2}$, ALBERTO CARO M. ${ }^{3}$ \\ 1. Médico Cirujano, Profesor Asociado, Facultad de Medicina, Universidad Andrés Bello. \\ 2. Estudiante, sexto año Medicina, Universidad Andrés Bello, Viña del Mar. \\ 3. Estadístico, Escuela de Medicina, Universidad Andrés Bello, Viña del Mar.
}

\begin{abstract}
Comparison of body temperature measurements obtained with otic digital thermometer and mercury axillary and rectal thermometers in children under the age of five

Background: In recent years, otic thermometers to measure body temperature in children have become increasingly popular as they reflect changes in body temperature sooner than other thermometers. The have become valuable assets in hospital emergency rooms; however, their accuracy and reliability need further studies. Objective: To compare corporal temperature readings between an otic thermometer and mercury axillary and rectal thermometers. Patients and Method: 50 patients under the age of 5, who were treated at the emergency room of our hospital, were randomly chosen to participate in this study. Temperature was measured on their right and left ears using an otic thermometer, and their rectal and right axillary temperature was taken using mercury thermometers. Patients were separated into 2 subgroups for comparison, children under and over 6 months old. Results: The results between the 2 subgroups were very similar. The average temperature obtained by rectal thermometers was $0.414^{\circ} \mathrm{C}$ higher than the average right-ear temperature $(\mathrm{p}<0.001)$ and $0.438^{\circ} \mathrm{c}$ higher than the average left-ear temperature $(\mathrm{p}<0.001)$. Conclusion: The otic thermometer's reading is significantly lower than the one obtained using mercury rectal and axillary thermometers.

(Key words: Thermometers, body temperature, fever, digital thermometer).

Rev Chil Pediatr 2013; 84 (3): 293-299
\end{abstract}

\section{RESUMEN}

Introducción: En los últimos años han aparecido nuevos métodos para medir la temperatura en niños, como el termómetro digital ótico. Siendo este un método más rápido para medir la temperatura y por lo tanto ideal para el servicio de urgencias, es necesario conocer su confiabilidad. Objetivo: Comparar los valores de temperatura corporal que entrega el termómetro ótico digital con los termómetros de mercurio axilar y rectal. Pacientes y Método: Se efectuó la medición de la temperatura en 50 pacientes menores de 5 años elegidos al azar, que con-

Recibido el 16 de noviembre de 2011, devuelto para corregir el 21 de marzo de 2012, segunda versión 18 de octubre de 2012 , aceptado para publicación el 06 de febrero de 2013.

Este trabajo cumple con los requisitos sobre consentimiento /asentimiento informado, comité de ética, financiamiento, estudios animales y sobre la ausencia de conflictos de intereses según corresponda.

Correspondencia a:

Francisca Soto-Aguilar B.

E-mail: fransotoaguilar@hotmail.com 
sultaron en el Servicio de Urgencia del Hospital de Niños y Cunas de Viña del Mar. Se les midió la temperatura con un termómetro digital ótico al lado izquierdo y derecho, y las temperaturas axilar derecha y rectal con termómetros de mercurio. EL análisis de los resultados se efectuó en el grupo estudiado, separado en dos grupos, menores y mayores de 6 meses. Resultados: Los resultados entre ambos subgrupos fueron los mismos. La temperatura media rectal obtenida fue $0,414^{\circ} \mathrm{C}$ más alta que la media ótica derecha $(\mathrm{p}<0,001)$ y $0,438^{\circ} \mathrm{C}$ más alta que la temperatura media ótica izquierda $(\mathrm{p}<0,001)$. Conclusión: El termómetro ótico digital entrega una temperatura significativamente más baja que la que entregan los termómetros de mercurio axilar y rectal. (Palabras clave: Termómetros, temperatura corporal, fiebre, termómetro digital).

Rev Chil Pediatr 2013; 84 (3): 293-299

\section{Introducción}

En los últimos años han aparecido nuevos métodos para tomar la temperatura en niños, uno de ellos es el termómetro digital ótico. Este termómetro tiene muchas ventajas en relación a los de mercurio, entre ellas destaca el corto tiempo de medición, fácil uso, mínima cooperación por parte del paciente y menos riesgos para los mismos ${ }^{1,2}$. La finalidad del estudio es aportar evidencia útil a los servicios de urgencia pediátricos, de manera que estos puedan conocer las limitaciones de la medición de temperatura ótica digital.

Desde el año 2010, los centros de salud públicos en Chile comenzaron un plan para abandonar el uso de instrumental con mercurio debido a la toxicidad de este material. Por otro lado, dado que en el Servicio de Urgencias los tiempos de atención son reducidos, lo ideal es contar con un método de medición de temperatura que sea rápido y fiable.

A pesar de las ventajas de los termómetros digitales, en la literatura aún es discutida su confiabilidad, por lo que es cuestionable si realmente podemos tomar decisiones clínicas en nuestros pacientes basándonos en esta medición.

Algunas fuentes apoyan el uso del termómetro digital ótico; lo califican como fiable y comparable a la toma con los termómetros de mercurio rectal, incluso ofreciéndolo como segunda opción en casos de que por alguna patología, este no se pudiera utilizar ${ }^{1,2}$, y se sugiere su utilización sobre los 6 meses de edad. Sin embargo, existen estudios que lo califican como un método de poca precisión y exacti$\operatorname{tud}^{3,4}$.

La hipótesis del presente estudio fue que los valores obtenidos mediante el termómetro ótico digital no son equivalentes a los valores obtenidos mediante los termómetros de mercurio en lactantes de edad menor o igual a 6 meses, a diferencia de los lactantes mayores a 6 meses. El objetivo de este estudio fue comparar los valores de temperatura corporal que entrega el termómetro ótico digital con los termómetros de mercurio axilar y rectal.

\section{Pacientes y Método}

Se evaluó con una población de 50 pacientes. El tamaño del grupo de estudio se calculó considerando el análisis de diferencia de medias para muestras pareadas. Los datos preliminares señalan que la diferencia en los resultados de temperaturas para muestras pareadas se distribuye normalmente con desviación estándar de $0,5^{\circ}$. Si la verdadera diferencia en las medias de las temperaturas de las muestras pareadas es $0,23^{\circ}$, se requiere una muestra de 50 pares de sujetos para rechazar la hipótesis nula que la diferencia en la respuesta media es cero grados con probabilidad (potencia) 0,9.

El error tipo I asociado con el test de hipótesis nula es 0,05 .

\section{Reclutamiento}

La muestra se obtuvo durante 7 días no consecutivos del mes de mayo del año 2011. El criterio de inclusión fue tener una edad menor a 5 años, y los pacientes se seleccionaron al azar a partir de la lista de espera de atención a medida que llegaban a consultar al Servicio de Urgencias del Hospital de Niños y Cunas de Viña del Mar. Se conversó con cada uno de los adultos que acompañaban a los pacientes, explicándoles lo que se iba a hacer y con qué pro- 
pósito, obteniendo su consentimiento verbal. Dos examinadores previamente entrenados en la técnica efectuaron las cuatro mediciones de temperatura; ótica izquierda, ótica derecha, axilar derecha y rectal; además de consignar la edad en meses y sexo de cada paciente.

\section{Instrumento y medición}

Para las mediciones se utilizaron 2 termómetros axilares de mercurio con bulbo largo, 2 termómetros rectales de mercurio con bulbo corto y 1 termómetro ótico digital (Braun $\left.{ }^{\circledR}\right)$. La precisión para rango de temperatura mostrado de este último termómetro es de $36^{\circ} \mathrm{C}$ a $39^{\circ} \mathrm{C}$ con un máximo error de laboratorio de $\pm 0,2^{\circ} \mathrm{C}$ dentro de este rango, $\mathrm{y} \mathrm{de} \pm 0,3^{\circ} \mathrm{C}$ fuera de este rango.

La técnica utilizada para medir la temperatura ótica consistió en traccionar el pabellón auricular hacia atrás en el caso de los lactantes, y hacia arriba y atrás en los niños mayores ${ }^{5}$. Se introdujo de esta forma el termómetro en el conducto auditivo externo, y se presionó el botón activador hasta que el aparato sonara avisando que se había obtenido el valor.

La temperatura axilar se midió en la axila derecha en todos los pacientes. Se comprobó que la axila del paciente estuviera seca y se agitó el termómetro hasta bajar la temperatura bajo $35^{\circ} \mathrm{C}$, se posicionó el bulbo en el centro de la axila. Se esperó $5 \mathrm{~min}^{6}$, se retiró el termómetro y se constató el valor obtenido.

Por último, la temperatura rectal se midió con el paciente acostado en prono sobre la falda del examinador, se agitó el termómetro hasta bajar el mercurio a menos de $35^{\circ} \mathrm{C}$, y luego se aplicó vaselina en el bulbo y se introdujo el mismo $2,5 \mathrm{~cm}$ al interior del recto durante $3 \mathrm{~min}^{6}$.

Todos los valores fueron interpretados por los mismos 2 examinadores, para disminuir la subjetividad de la lectura. Para la desinfección de los termómetros, se utilizó solución antiséptica de clorhexidina y alcohol entre la medición de cada paciente.

Análisis estadístico. Se utilizó el programa SPSS (versión 15). Para verificar el supuesto de normalidad de las mediciones se empleó la prueba de Kolmogorov-Smirnov. Para comparar las diferencias entre las diversas mediciones se aplicó la prueba de diferencia de medias para muestras relacionadas. Por último, se utilizó la prueba $t$ para diferencia de medias poblacionales relacionadas para comparar los resultados de pacientes de 6 meses de edad o menos, con los pacientes mayores a 6 meses de edad. En todas las pruebas se usó el valor $p<0,05$ como nivel de significación estadística.

\section{Resultados}

Las tendencias en el registro de temperatura ótica, axilar y rectal fueron semejantes (tabla 1), las correlaciones entre las mediciones resultan significativas $(p<0,001)$ (tabla 2$)$. En todos los casos se acepta la hipótesis nula que la muestra proviene de una población con distribución normal $(\mathrm{p}>0,05)$.

Como se describe en la tabla 3, la hipótesis nula de que las medias de las temperaturas de las dos poblaciones que se comparan son iguales, se rechaza en las comparaciones de las parejas 3,5 y $6(\mathrm{p}<0,001)$. Por lo tanto, resulta significativamente mayor la temperatura media rectal en comparación con la temperatura media ótica derecha $\left(0,414^{\circ} \mathrm{C}\right.$ con $\left.\mathrm{p}<0,001\right)$, la temperatura media rectal en comparación con la temperatura media ótica izquierda $\left(0,438^{\circ} \mathrm{C}\right.$ con $\mathrm{p}<0,001)$, y la temperatura media rectal en comparación con la temperatura media axi$\operatorname{lar}\left(0,506^{\circ} \mathrm{C}\right.$ con $\left.\mathrm{p}<0,001\right)$.

La distribución de edad de la muestra correspondió en un $24 \%$ a pacientes menores o iguales a 6 meses de edad, y un $76 \%$ a pacientes mayores de 6 meses de edad. En las tablas 4 y 5 se muestran las temperaturas de estos dos grupos por separado. La prueba t para diferencia de medias poblacionales relacionadas de-

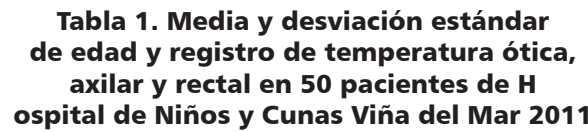

\begin{tabular}{|lccc|}
\hline Variables & Media & Desviación típica & n \\
\hline Edad (meses) & 24,22 & 17,591 & 50 \\
\hline To ótica der & 37,22 & 0,786 & 50 \\
\hline To ótica izq & 37,20 & 0,686 & 50 \\
\hline To axilar & 37,13 & 0,937 & 50 \\
\hline To rectal & 37,64 & 0,878 & 50 \\
\hline
\end{tabular}

Elaboración propia. 
Tabla 2. Correlación y significancia de muestras relacionadas en $\mathbf{5 0}$ pacientes de Hospital de Niños y Cunas Viña del Mar 2011

\begin{tabular}{|llccc|}
\hline \multicolumn{1}{|c}{ Pares variables } & n & Correlación & p \\
\hline Par 1: & To otica der - To otica izq & 50 & 0,871 & $<0,001$ \\
\hline Par 2: & T$^{\circ}$ otica der - To axilar & 50 & 0,798 & $<0,001$ \\
Par 3: & To otica der - To rectal & 50 & 0,775 & $<0,001$ \\
Par 4: & To otica izq - To axilar & 50 & 0,876 & $<0,001$ \\
\hline Par 5: & To otica izq - To rectal & 50 & 0,824 & $<0,001$ \\
Par 6: & To axilar - To rectal & 50 & 0,891 & $<0,001$ \\
\hline
\end{tabular}

Elaboración propia. mostró diferencia significativa para pares 3,5 y $6(\mathrm{p}<0,001)$ en la población menor o igual a 6 meses de edad. Entre temperatura axilar y rectal hubo una diferencia de $0,725^{\circ}$ que es significativa $(\mathrm{p}<0,001)$ (tabla 4).

También se demostró una diferencia significativa para pares 3,5 y $6(\mathrm{p}<0,001)$ en la población mayor a 6 meses de edad, y entre la temperatura axilar y la rectal hubo una diferencia de $0,437^{\circ}$ que es significativa $(\mathrm{p}<0,001)($ tabla 5$)$.

Tabla 3. Distribución de diferencias de media y desviación estándar de temperatura corporal, según forma de medición

\begin{tabular}{|c|c|c|c|c|c|}
\hline & \multirow[t]{2}{*}{ Pares variables } & \multicolumn{2}{|c|}{ Diferencias relacionadas } & \multirow[t]{2}{*}{$\mathbf{T}$} & \multirow[t]{2}{*}{ Sig (bilateral) } \\
\hline & & Media & Desviación típica & & \\
\hline Par 1: & $\mathrm{T}^{\circ}$ ótica der - $\mathrm{T}^{\circ}$ ótica izq & 0,02 & 0,3863 & 0,439 & 0,662 \\
\hline Par 2: & $\mathrm{T}^{\circ}$ ótica der - $\mathrm{T}^{\circ}$ axilar & 0,09 & 0,5663 & 1,149 & 0,256 \\
\hline Par 3: & $\mathrm{T}^{\circ}$ ótica der - To rectal & $-0,41$ & 0,5646 & $-5,185$ & $<0,001$ \\
\hline Par 4: & $\mathrm{T}^{\circ}$ ótica izq - To axilar & 0,07 & 0,4718 & 1,019 & 0,313 \\
\hline Par 5: & To ótica izq - To rectal & $-0,44$ & 0,4993 & $-6,202$ & $<0,001$ \\
\hline Par 6: & $\mathrm{T}^{\circ}$ axilar - $\mathrm{T}^{\circ}$ rectal & $-0,51$ & 0,4273 & $-8,373$ & $<0,001$ \\
\hline
\end{tabular}

Elaboración propia.

Tabla 4. Distribución de diferencias de media y desviación estándar de temperatura corporal, según forma de medición en $\leq 6$ meses de edad

\begin{tabular}{|c|c|c|c|c|c|}
\hline & \multirow[t]{2}{*}{ Pares variables } & \multicolumn{2}{|c|}{ Diferencias relacionadas } & \multirow[t]{2}{*}{$\mathbf{T}$} & \multirow[t]{2}{*}{ Sig (bilateral) } \\
\hline & & Media & Desviación típica & & \\
\hline Par 1: & To ótica der - $\mathrm{T}^{\circ}$ otica izq & 0,03 & 0,3596 & 0,241 & 0,814 \\
\hline Par 2: & $\mathrm{T}^{\circ}$ ótica der - $\mathrm{T}^{\circ}$ axilar & 0,10 & 0,5009 & 0,692 & 0,504 \\
\hline Par 3: & $\mathrm{T}^{\circ}$ ótica der - $\mathrm{T}^{\circ}$ rectal & $-0,63$ & 0,2179 & $-9,934$ & $<0,001$ \\
\hline Par 4: & $\mathrm{T}^{\circ}$ ótica izq - $\mathrm{T}^{\circ}$ axilar & 0,08 & 0,3279 & 0,792 & 0,445 \\
\hline Par 5: & $\mathrm{T}^{\circ}$ ótica izq - $\mathrm{T}^{\circ}$ rectal & $-0,65$ & 0,1931 & $-11,663$ & $<0,001$ \\
\hline Par 6: & To axilar - To rectal & $-0,73$ & 0,3494 & $-7,189$ & $<0,001$ \\
\hline
\end{tabular}

Elaboración propia.

Tabla 5. Distribución de diferencias de media y desviación estándar de temperatura corporal, según forma de medición en $>6$ meses de edad

\begin{tabular}{|c|c|c|c|c|c|}
\hline & \multirow[t]{2}{*}{ Pares variables } & \multicolumn{2}{|c|}{ Diferencias relacionadas } & \multirow[t]{2}{*}{$\mathbf{T}$} & \multirow[t]{2}{*}{ Sig (bilateral) } \\
\hline & & Media & Desviación típica & & \\
\hline Par 1: & $\mathrm{T}^{\circ}$ ótica der - $\mathrm{T}^{\circ}$ otica izq & 0,02 & 0,3989 & 0,366 & 0,716 \\
\hline Par 2: & $\mathrm{T}^{\circ}$ ótica der - $\mathrm{T}^{\circ}$ axilar & 0,09 & 0,5917 & 0,932 & 0,357 \\
\hline Par 3: & T० ótica der - $\mathrm{T}^{\circ}$ rectal & $-0,35$ & 0,6237 & $-3,433$ & $<0,001$ \\
\hline Par 4: & $\mathrm{T}^{\circ}$ ótica izq - $\mathrm{T}^{\circ}$ axilar & 0,07 & 0,5127 & 0,791 & 0,434 \\
\hline Par 5: & To ótica izq - To rectal & $-0,37$ & 0,5477 & $-4,176$ & $<0,001$ \\
\hline Par 6: & $\mathrm{T}^{\circ}$ axilar - $\mathrm{T}^{\mathrm{o}}$ rectal & $-0,44$ & 0,4302 & $-6,259$ & $<0,001$ \\
\hline
\end{tabular}

Elaboración propia. 
Así, las diferencias entre los valores obtenidos mediante los distintos métodos se mantienen para mayores de 6 meses y para menores de 6 meses.

\section{Discusión}

Los lugares más apropiados para medir la temperatura corporal son los que están más cerca al hipotálamo, el centro regulador de temperatura que refleja la temperatura central $^{7}$. Como el hipotálamo es inaccesible, la temperatura central generalmente se mide en la arteria pulmonar, esófago distal, vejiga y nasofaringe ${ }^{8,9}$. Estos métodos son invasivos, y por lo tanto no se pueden utilizar para medir las temperaturas en los servicios de urgencias; los instrumentos para efectuar la medicón de la temperatura deben ser no invasivos, accesibles y rápidos, y los que más se usan son los termómetros digitales (óticos, axilares y rectales) y los de mercurio (axilares o rectales). La medición de la temperatura, presenta variaciones dependiendo de la zona donde se mida, y entre ellos el criterio de referencia descrito para la medición de la temperatura corporal es la rectal, siendo la más exacta en todas las edades $^{10,11}$. Considerando que la fiebre es definida de manera exacta y precisa como: temperatura Rectal $\geq 38^{\circ} \mathrm{C}$, Axilar $\geq 37,5^{\circ} \mathrm{C}$ y Ótica $\geq 38^{\circ} \mathrm{C}$; es de vital importancia saber si los valores de la medición en cuestión son realmente confiables ${ }^{1}$.

La temperatura axilar es considerada menos precisa, sin embargo puede ser útil como primera aproximación en mayores de 3 meses en caso de que no se pueda tomar la temperatura rectal ${ }^{1}$. Es considerada menos confiable específicamente porque se han realizado estudios termográficos que han mostrado que una vez que la axila del paciente se abre para permitir la adecuada colocación del termómetro, se produce una caída en la temperatura. Este descenso se podría recuperar manteniendo el termómetro por 10 min en el sitio de medición ${ }^{2}$, sin embargo, en el presente estudio se mantuvo el termómetro por 5 min para dar cumplimiento a la técnica de medición seleccionada ${ }^{6}$. Si bien esto pudo haber alterado los resultados, se considera normal que exista una variación entre la temperatura axilar y rectal dentro de un rango entre $0,5^{\circ} \mathrm{C}$ y $1,2^{\circ} \mathrm{C}^{1,10,11}$, diferencia que se obtuvo en los resultados de este estudio, lo que estaría demostrando que la técnica para medir tanto la temperatura rectal como axilar fueron correctamente realizadas.

La temperatura ótica mide la cantidad de calor producida por la arteria tempora ${ }^{11}$, detectando la radiación térmica. Es importante considerar que la medición se puede ver alterada por diversos factores, entre ellos, si el paciente ha estado recostado sobre el pabellón auricular en el que se realizará la toma de temperatura, si se encuentra llorando en el momento de la medición, si ha estado expuesto al frío o al calor, o si el termómetro se posicionó adecuadamente en el canal auditivo, lo que no es siempre posible dependiendo del tamaño del canal auditivo del paciente, que se modifica con la edad $^{5,7}$. Este último factor es probablemente el motivo por el cual no se recomienda el uso de este termómetro en menores de 6 meses.

Dado que la temperatura ótica debiese ser la misma que la rectal ${ }^{4}$, sería una forma más fácil de obtener la temperatura corporal, considerando además que el termómetro digital utilizado para medir la primera es más rápido. Los resultados de este estudio muestran una diferencia significativa entre ambos, que se podría explicar por los factores ya mencionados que pueden alterar la toma de temperatura ótica $^{12,14}$. Sin embargo, este resultado apoya nuestra hipótesis de que los valores obtenidos mediante el termómetro ótico digital no son equivalentes con los valores obtenidos mediante los termómetros de mercurio. Existen estudios publicados previamente que también apoyan esta hipótesis, que concluyen que esta medición no se puede utilizar para la detección de la fiebre en la toma de decisiones clínicas ${ }^{11}$. Cabe destacar que este estudio se realizó para evaluar los métodos usados para medir la temperatura corporal en servicios de urgencia, que no necesariamente es lo mismo que la temperatura que se mide fuera del hospital por personas no capacitadas; en este escenario la dificultad del uso e interpretación del termómetro de mercurio rectal son suficientes para no recomendarlo ${ }^{15}$.

Por último, al comparar los valores de tem- 
peratura obtenidos en el grupo de pacientes de 6 meses o menos con el de pacientes mayores de 6 meses (tablas 4 y 5), no se encontró una diferencia significativa entre ellos. Este análisis se efectuó con un número de 12 pacientes de 6 meses o menos y 38 pacientes mayores de 6 meses, pudiendo ser esto una limitación, por no ser un número suficiente de pacientes para cada grupo.

La diferencia anatómica del conducto auditivo externo de los menores de 6 meses en comparación con los mayores, razón por la que se decidió separar estos subgrupos para las comparaciones en este estudio, parece no alterar significativamente los resultados si se utiliza una buena técnica de toma de temperatura. Con este resultado se rechaza parcialmente la hipótesis de este trabajo.

\section{Conclusiones}

Los valores obtenidos mediante el termómetro ótico digital no son equivalentes con los valores obtenidos mediante los termómetros de mercurio. Esto se cumple tanto para los pacientes de edad menor o igual a 6 meses, como para pacientes mayores de 6 meses que entraron en este estudio, con las mismas diferencias.

Es cuestionable, por lo tanto, tomar decisiones clínicas sobre la base de la medición de la temperatura corporal medida con un termómetro ótico digital. Considerando la ventaja que tiene este último por no tener mercurio $\mathrm{y}$ por ser más rápido, habría que ser más cautelosos en la técnica de medición, y tal vez en casos en que la toma de decisiones importantes se base en la presencia o no de fiebre, considerar el uso de un termómetro de mercurio es una buena alternativa. Cuando se desee utilizar el termómetro de mercurio, si no se pueda tomar la temperatura rectal a los pacientes, se recomienda como alternativa tomar la temperatura axilar con este instrumento.

Sobre la base de los métodos de medición de temperatura, utilizados en el presente estudio, el criterio de referencia para medir la temperatura corporal en todas las edades sigue siendo la técnica de toma de temperatura rectal con termómetro de mercurio.

\section{Agradecimientos}

Agradecemos al Servicio de Urgencia del Hospital de Niños y Cunas de Viña del Mar, sin su apoyo no habría sido posible realizar el estudio de manera exitosa.

\section{Referencias}

1.- Baraff LJ: Management of fever without source in infants and children. Ann Emerg Med 2000; 36: 602.

2.- Saxena AK, Topp S, Heinecke A, Willital GH: Application criteria for infrared ear thermometers in pediatric surgery. Technology and Health Care 2001; 9: 281-5.

3.- Craig JV, Lancaster GA, Taylor S, Williamson PR, Smyth $R L$ : Infrared ear thermometry compared with rectal thermometry in children: a systematic review. Lancet 2002; 360: 603.

4.- Lawson L, Bridges EJ, Ballou I, Efaker R, Greco S: Accuracy and precision of noninvasive temperature measurement in adult intensive care patients. Am J Crit Care 2007; 16: 484-96.

5.- Guraieb R: Estudio comparativo de diferentes métodos para tomar la temperatura en los niños. Anales Médicos Hospital ABC 1997; 42: 18-22.

6.- Children's Institute for Pediatric Education: Temperature: Oral, Rectal and Axillary Using a Glass Thermometer. Copyright 1975-2005, Children's Hospital, Inc., Columbus, Ohio.

7.- Schmitz T, Blair N, Falk M, Levine C: A comparison of five methods of temperature measurement in febril intensive care patients. Am J Crit Care 1995; 4: 28692.

8.- Milewski A, Ferguson K, Terndrup T: Comparison of pulmonary artery, rectal and tympanic membrane temperatures in adult intensive care unit patients. Clin Paediatr 1991; 30: 13-6.

9.- El-Randhi A, Barry $W$ : Thermometry in paediatric practice. Arch Dis Child 2006; 91: 351-6.

10.- Bates $B$ : A guide to physical examination and history taking, 6th ed, Lippincott, Philadelphia 1995.

11.- Paes BF, Vermeulen K, Brohet RM, et al: Accuracy of tympanic and infrared skin thermometers in children. Arch Dis Child 2010; 95: 974.

12.- Erickson RS, Woo TM: Accuracy of infrared ear thermometry and traditional temperature methods in young children. Heart Lung 1994; 23: 181-95.

13.- McCaffrey T, McCook R, Wurster R: Effect of head skin temperature on tympanic and oral temperature in man. $\mathrm{J}$ 
Appl Physiol 1975; 39: 114-8.

14.- Bridges E, Thomas K: Noninvasive Measurement of Body Temperature in Critically III Patients. Crit Care Nurse 2009; 29: 94-7.

15.- Oncel MY, Tekgunduz KS, Ozdemir R, et al: A Compari- son of Different Methods of Temperature Measurement by Mothers and Physicians in Healthy Newborns. Indian J Pediatr, 2012 Jun 2 [Epub ahead of print] disponible en http://www.ncbi.nlm.nih.gov/pubmed/22660904, última vista 29-07-2012. 